\title{
Text repetition and text integration
}

\author{
W. Matthew Collins and Betty Ann Levy \\ McMaster University, Hamilton, Ontario, Canada
}

\begin{abstract}
Two experiments explored the levels of text representation that mediate text repetition effects, following the Raney (2003) model. The magnitude of the repetition benefit in Experiment 1 supported predictions of Raney's model, indicating that the ease of forming a situation model contributed to the magnitude of the reprocessing benefit. In addition, representations organized around a good situation model were more sensitive to changes than were representations formed from reading without a good situation model. The results of Experiment 2 did not support the suggestion that the surface form and textbase are bound to a well-developed situation model, thereby limiting repetition effects to similar linguistic contexts. Rather, the nature of the repetition benefits in the present series of experiments are better explained by the degree of overlap between passages at each of the three levels of text representation.
\end{abstract}

For both fluent adult readers and beginning readers in elementary school, repeated reading of the same text leads to faster and more accurate reading, with no loss in comprehension (for reviews, see Levy, 1993, 2001; Raney, 2003; Tenpenny, 1995). The theoretically interesting issue involves the nature of the memory representations formed during reading that can be recruited in the service of more fluent reprocessing on a later occasion. That is, what kinds of memory representations lead to practice or repetition benefits in text processing? Although the repetition benefit itself is well established, considerable debate has arisen regarding the nature of the memorial representations involved.

In the earlier literature, there were two main theoretical positions involved in this debate. According to the $a b$ stractionist position, the repetition benefit resulted from reprocessing lexical units. That is, lexical representations of individual word units were primed during the first reading, so that later rereading of these units was faster and more accurate. Carr and his associates were the main advocates of this position (Brown \& Carr, 1993; Carlson, Alejano, \& Carr, 1991; Carr \& Brown, 1990; Carr, Brown, \& Charalambous, 1989). Carr et al. asked participants to read and reread aloud paragraphs that were in normal form or were scrambled word versions of the paragraphs. The basic finding was that there were equivalent benefits in rereading time whether the same or different versions were read on both occasions. That is, if a reader first read a scrambled word version and then reread a normal version, the repetition benefit was equal to when a normal version was read twice. These data clearly supported the view that the repetition benefit was at the single-word level, since loss of all of the text level structure in the scrambled paragraphs led to no loss in the repetition effect. Furthermore, Carr et al. found complete transfer across variations in font, suggesting that the lexical representations were abstract, carrying no information about the features of the visual input. These data offered strong support for the abstractionist explanation of the text repetition benefit.

However, Levy and her colleagues found results quite different from those of the Carr group (Levy \& Burns, 1990; Levy et al., 1995; Levy, DiPersio, \& Hollingshead, 1992; Levy \& Kirsner, 1989). Levy and Burns asked participants to silently read and then reread texts that varied in form, following Carr et al.'s (1989) paradigm. However, they varied the level at which the scrambling occurred (paragraph, sentence, and word levels). They found that when a normal text was reread, there was a loss in the repetition benefit as the structure of the text version read first decreased. When the scrambling was at the word level, no transfer benefit was observed, failing to replicate Carr et al.'s findings. Levy, Masson, and Zoubeck (1991) also reported no transfer benefit from reading wordscrambled to reading normal texts. These data indicated that the memorial representations retained information about the higher order linguistic structure of the text and this information mediated the magnitude of the transfer benefit from original to rereading occasions. Levy and her colleagues argued for an episodic form of text representation. They suggested that texts were represented in a holistic fashion and that recruitment of the reading episode was necessary for the lower level representations to be recruited to aid in the repetition benefit.

More recently, Raney (2003) has proposed an alternative to the abstract-episodic dichotomy. Raney's contextdependent representation model attempts to account for evidence from both abstract and episodic accounts of text repetition by incorporating ideas from van Dijk and Kintsch's model (1983). The van Dijk and Kintsch model postulated three levels of representation: The surface-

W. M. Collins, collinwm@mcmaster.ca 
level representations consist of the wording used in the text and are formed by processing lexical and syntactic information; the text proposition representations are formed from the meaning analyses of the text statements; and the situation model representations combine the text-based information with prior knowledge. This level represents the events and actions (episodes) described in the text, with text content combined with inferences and general knowledge used to comprehend the text's message.

Raney's (2003) context-dependent model builds upon van Dijk and Kintsch's (1983) model by proposing that the three levels of representation reflect different degrees of context dependence. According to the model, the surface form and textbase are represented in a context-independent manner, and a well-developed situation model binds together the surface form and the textbase, thereby creating a context-dependent representation. Raney argued that a reader always attempts to develop some type of situation model. The ease of developing a situation model depends on a number of factors, including task demands (e.g., instructions), processing strategies (e.g., reading for meaning), characteristics of the text (e.g., text coherence), and reader characteristics (e.g., reading ability). Establishing a well-developed situation model leads to context-dependent representations, which in turn leads to context-dependent repetition effects.

Raney's (2003) model makes two predictions in regard to the nature of context-dependent text representations and subsequent transfer across reading episodes. On the one hand, Raney proposed that it is the degree of overlap at each level of representation that determines the magnitude of the repetition benefit between passages. The greater the overlap between the surface form, textbase, and situation model of two passages, the larger the expected repetition benefit between them. Raney referred to this as a contextdependent continuum. On the other hand, Raney also suggested that when a well-developed situation model is established, it binds the surface form and the textbase to the text representation, creating a tightly integrated memory structure that produces repetition benefits only when the same or a similar situation model is reinstated. We will refer to this as the strong prediction of Raney's model. According to the strong prediction of the model, if only the surface form and the textbase are reinstated in the context of different situation models, there will be little to no repetition benefit, because both levels of memory representations are bound to the previous reading episode by the situation model, consistent with the results of Levy and her colleagues (Levy \& Burns, 1990; Levy et al., 1995). Alternatively, if there is no situation model or a poorly developed situation model, the surface form and the textbase are not tightly bound to the text representation, and there can be repetition benefits on subsequent readings when the surface form and/or textbase are reinstated (Raney, 2003), consistent with the results of Carr et al. (1989).

A number of studies have confirmed Raney's (2003) predictions related to the combined effects of word and meaning levels on the magnitude of rereading transfer. Raney, Therriault, and Minkoff (2000) asked participants either to read identical versions of a passage on both oc- casions or to reread a paraphrased version of the original text. They reported a larger reprocessing advantage for identical than for paraphrased conditions. Since the same situation model was involved in both cases, the reduced benefit with changes in wording indicated a contribution to the total transfer from the lexical level. Furthermore, synonyms were reprocessed more quickly only during second fixations, whereas identical words were processed more quickly on both first and second fixations. Raney et al. (2000) suggested that second fixations index processing related to semantic integration, whereas first fixations also index word identification. Thus, identical words are identified more easily and integrated into the text more easily during rereading, whereas only the semantic integration phase is facilitated for synonyms. Again, both word level and text integration levels of processing combine to determine the magnitude of the rereading advantage.

Levy (2001) more directly examined the influence of a situation model on the magnitude of rereading transfer. Whereas Raney et al.'s (2000) experiment held the situation model constant and varied the word level, Levy (2001) held the surface and text proposition information constant across readings and varied only the ease of forming a situation model during reading. To do this, she used passages modeled after those described by Bransford (1979). These passages describe an everyday event, such as doing the laundry. Each sentence is completely coherent, but the referent event is ambiguous unless a title is given with the passage (e.g., washing clothes). Thus, when reading without a title, participants would have difficulty deriving a situation model to organize the sentences and paragraphs into a meaningful event sequence. When participants read with a title, however, text processing is facilitated, and the text representation should be contextually bound to the situation model.

University students read and then immediately reread Bransford-type passages. The presence or absence of a title on each occasion was manipulated. On the first reading, the presence of a title led to faster reading, replicating Bransford's (1979) study. The more critical finding was that the memory for the first reading influenced the magnitude of the reprocessing advantage. When the rereading text had a title (instantiating the correct situation model), rereading was always faster than first reading. However, the magnitude of this rereading benefit was considerably larger when the passage had also first been read with a title, as compared with when it had been read without a title. That is, the memory for the prior experience had a substantial influence on the magnitude of transfer, even when the passage being reread had a title that made it easy to understand. Note that in both the title and the no-title conditions, exactly the same surface information and text propositions were being read. A larger repetition benefit in the title condition reflects the benefit that comes from previously creating a memory representation bound to a well-developed situation model. In other words, when the text representation of a previously encountered passage was formed with a good situation model, it facilitated reading of the repetition passage over and above any facilitation due to the presence of a situation model alone at the rereading stage. Thus, consistent with the 
Raney (2003) model and Raney et al.'s (2000) experiment, repetition benefits in text processing were influenced by the episodic organization of the memory representation.

To date, very few studies have tested Raney's (2003) notion that a context-dependent representation is created under reading conditions in which a well-developed situation model is established. Raney, Minkoff, and Therriault (1997) found some support for the prediction that repetition effects are limited to similar linguistic contexts. The critical measure in their study was fixation duration when participants encountered the same target word in two interleaved texts. These texts shared some themes, while each also had unrelated themes. If the target word occurred in a shared theme, fixation duration was reduced on the second encounter with a word, but it was not reduced if the word occurred twice in the nonoverlapping themes. Raney et al. (1997) suggested that the overlapping themes constituted the same situation model and that words bonded to these themes should be reprocessed more quickly. This shows there is some contextual dependency of word-level transfer when words are processed with the same situation model.

The experiments to be reported here investigated the predictions of Raney's (2003) model regarding contextdependent text representations (text representations formed around a strong situation model). Experiment 1 examined Raney's proposal that representations bound to a situation model are context dependent. The question addressed was whether scrambling the sentences upon rereading would have a larger impact on texts that were originally organized around a situation model, as compared with texts that were not originally bound to a situation model. According to Raney's model, the representation formed by a situation model is context dependent. Consequently, any change to the textbase upon rereading should hinder recruitment of the original memory representation, and transfer should be impaired relative to conditions in which the textbase is left unchanged. In other words, scrambling the textbase during rereading leads to a mismatch between the organization of the memory traces and the organization of the text being read. This should lead to a loss of the transfer benefit. In contrast, scrambling the sentences of the rereading text should not have as great an impact when the original reading was with a weak situation model, because the memory representation of the text propositions would be context independent in this case.

Experiment 2 examined Raney's (2003) strong prediction that under reading conditions in which a welldeveloped situation model is established, the surface form and textbase are bound to the text representation. As in the Raney et al. (1997) study, we examined transfer between pairs of highly related passages and pairs of less related passages. However, unlike in the experiment of Raney et al. (1997), we examined whether processing was facilitated between portions of the textbase representation shared between passages. According to the strong prediction of Raney's model, processing should be facilitated only between highly related passages. When the passages are less related, the strong prediction of Raney's model is little or no facilitation, because the linguistic contexts are different and the situation model formed in the first reading will not be recruited when the sentences are reprocessed in the context of a different situation model.

\section{EXPERIMENT 1}

\section{Method}

Participants. The participants in this study were 48 undergraduate students from psychology classes at McMaster University. All the volunteers were native English speakers who received course credit for their participation.

Materials. The participants read 16 consecutive passages presented on a computer screen. These 16 passages consisted of eight different texts, each presented twice. All repetitions were after a delay, with four randomly selected texts having a lag of 2 intervening passages and the remaining four having a lag of 3 intervening passages. The two lags were randomly distributed across the 16 passages, so that the occurrence of a repetition could not be predicted.

Each of the eight different texts were 400 words in length; they appeared on 34 lines, left and right justified, on the computer screen. All the passages were descriptive narratives of everyday activities, such as taking an exam or shopping for shoes. The passages were written to conform to the style first reported by Bransford (1979). In this style, each individual sentence is coherent and meaningful, but the overall referent event that is described by the sentences is ambiguous unless the passage is accompanied by a title. The title provides the referent event around which the passage can be organized into a meaningful event sequence. Appendix A contains a normal and a scrambled version of a sample passage and the comprehension questions for that passage.

The passages were presented line by line on the computer screen. The participants pressed the space bar to begin reading each passage. The first keypress brought either the title of the passage or a row of Xs to the top of the screen. The next keypress led to the presentation of the first line of text. Each keypress thereafter led to the presentation of another line of text immediately below the previous line. All the lines of the text remained on the screen until the screen was full. The next keypress then began a new screen (as if the reader had turned the page in a book), and the new line of text was at the top of the new page. Each subsequent keypress then presented another line of text immediately below the previous line until the passage ended. This presentation of the text modeled reading pages of a book, where text on a page remains visible to allow regressive eye movements while reading.

Reading time for each passage was recorded by the computer. Timing of the passage started with the keypress to bring up the first line of the text. Timing of the passage stopped with the keypress to remove the last line of the text. In order to ensure that the participants were reading for meaning, the participants answered three comprehension questions for each passage after all 16 passages had been read. The three questions per story were asked together, and the order of question triads mirrored the first occurrence of the stories in the passage set. The questions were open-ended and asked about facts that were explicitly stated in the story. The first line of a passage preceded the three questions for that passage, so as to cue the particular text being queried. Questions were presented in a booklet, and the participants wrote their responses in the booklet.

Procedure. The participants were tested in individual sessions lasting approximately $1 \mathrm{~h}$. They were instructed to read quickly but with comprehension so that they could answer the comprehension questions. Before beginning the experimental passages, the participants were given a practice passage to familiarize them with the line-by-line presentation format and keypress responding.

Design. There were two experimental manipulations. One manipulation was the presence (T) or absence (NT) of a title during both readings. The second manipulation concerned the organization of the text on the second reading; texts either were presented normally or were scrambled (S) at the sentence level. Texts were always or- 
ganized normally on the first reading. Four conditions were created by these manipulations: title present on both readings, with normal text on the second reading $(\mathrm{T}-\mathrm{T})$; no title present on both readings, with normal text on the second reading (NT-NT); title present on both readings, but with scrambled sentences on the second reading (T-TS); and finally, no title present on both readings, but with scrambled sentences on the second reading (NT-NTS). The participants were presented all eight stories twice each, with two stories appearing in each condition. Across participants, each of the eight stories appeared in all four conditions equally often.

Reading times were first analyzed using two series of paired sample $t$ tests. The first series of $t$ tests were conducted to establish that the title manipulation affected reading times in a manner consistent with the idea that providing a situation model leads to more fluent reading. The second series of $t$ tests examined whether there was a significant effect of repetition in each of the four conditions. The first readings did not include a scrambled condition, and thus we did not use a three-way ANOVA.

In order to examine the magnitude of the repetition effect in each of the four different conditions (T-T, NT-NT, T-TS, and NT-NTS), difference scores were computed between first and second readings. Thus, in each condition, the mean overall reading time for the repetition was subtracted from the mean overall reading time on the first reading. We then compared the repetition benefits in $\mathrm{T}-\mathrm{T}$ and NT-NT conditions, using a paired sample $t$ test. This comparison tests the advantage of reading and rereading with an intact situation model, and if the results of Levy (2001) are replicated, there should be a significantly larger repetition benefit in the $\mathrm{T}-\mathrm{T}$ condition than in the NT-NT condition.

Lastly, we computed a 2 (title or no title) $\times 2$ (text: normal or scrambled) repeated measures ANOVA on the difference scores to examine the effects of scrambling the textbase during rereading. According to Raney's (2003) model, when a representation is contextually bound to the situation model (title condition), there should be a smaller repetition benefit if the textbase is changed upon rereading. On the contrary, when a representation is not strongly bound to the situation model (no-title condition), the repetition should not be greatly affected by a change to the textbase. Thus, we expected to find an interaction between title and text.

\section{Results and Discussion}

Analyses of overall reading times revealed a significant effect of title $[t(96)=-4.01, p<.01$, for first reading, and $t(96)=-4.57, p<.01$, for second reading]. Thus, passages with a title were read more quickly than passages without a title on both repetitions, regardless of whether they were scrambled or normal. This is the benefit to processing when the passage is more comprehensible. In addition, there was a significant effect of repetition, since second-reading times were always significantly shorter than first-reading times across all conditions $[\mathrm{T}-\mathrm{T}, t(47)=8.35, p<.01 ; \mathrm{NT}-\mathrm{NT}, t(47)=5.92, p<$ .01 ; $-\mathrm{TS}, t(47)=4.55, p<.01 ;$ and NT-NTS, $t(47)=$ 7.12, $p<.01]$.

A paired samples $t$ test comparing the magnitude of the repetition effect for the $\mathrm{T}-\mathrm{T}$ versus the $\mathrm{NT}-\mathrm{NT}$ conditions revealed a significant difference $[t(47)=2.14, p<.05]$. Thus, the presence of a title on both readings significantly increased the repetition effect, as compared with when both readings were without a title. This is consistent with Raney's (2003) proposal and the results of previous studies (Levy, 2001) that the presence of a situation model maximizes fluency across experiences.

Difference scores. A $2 \times 2$ repeated measures ANOVA on the difference scores revealed a significant interaction
Table 1

Mean Reading Times (in Milliseconds) With Standard Errors As a Function of Title, Organization of Text Upon Rereading, and Repetition, Experiment 1

\begin{tabular}{|c|c|c|c|c|c|c|}
\hline \multirow[b]{3}{*}{ Organization } & \multicolumn{4}{|c|}{ Repetition } & \multirow{2}{*}{\multicolumn{2}{|c|}{$\begin{array}{c}\text { Repetition } \\
\text { Benefit }\end{array}$}} \\
\hline & \multicolumn{2}{|c|}{ First } & \multicolumn{2}{|c|}{ Second } & & \\
\hline & $M$ & $S E$ & $M$ & $S E$ & $M$ & $S E$ \\
\hline \multicolumn{7}{|c|}{ Title Present } \\
\hline Normal upon rereading & 104 & 3 & 87 & 3 & 17 & 2 \\
\hline Scrambled upon rereading & 103 & 3 & 95 & 3 & 8 & 2 \\
\hline \multicolumn{7}{|c|}{ Title Absent } \\
\hline Normal upon rereading & 108 & 3 & 96 & 3 & 12 & 2 \\
\hline Scrambled upon rereading & 111 & 3 & 100 & 3 & 11 & 1 \\
\hline
\end{tabular}

between title and text $\left[F(1,47)=7.14, M S_{\mathrm{e}}=83.77, p<\right.$ $.01]$. As Table 1 shows, when the passage was read with a title on both readings, scrambling the textbase during rereading led to a significantly smaller repetition benefit $[t(47)=4.06, p<.001]$, as compared with when the textbase was normal (T-T vs. T-TS). However, when both readings were without a title, the repetition effect was unaffected by scrambling the text on the second reading $[\mathrm{NT}-\mathrm{NT}$ vs. NT-NTS, $t(47)=0.38, p>.05]$. Taken together, these results provide support for Raney's (2003) idea that representations bound by a situation model are context dependent. Raney predicted that because the representation formed with a strong situation model is context dependent, changing the textbase that is bound to the situation model should significantly impair the transfer benefit, because the situation model binds the textbase to a specific semantic organization. The smaller repetition benefit in the T-TS condition, relative to the T-T condition (context kept constant), indicates that scrambling the sentences did significantly reduce this benefit. In contrast, Raney's model suggests that scrambling the sentences of a text during rereading should not have as great an impact when the text is organized around a weak situation model (no title), because without the use of a well-formed situation model, the sentences are represented at the textpropositional level and this level is context independent. We also found support for this proposal; scrambling the sentences so that the textbase organization changed did not affect the repetition benefit in the NT-NTS condition, relative to the NT-NT condition.

Comprehension scores. The comprehension scores for texts in each condition were combined to yield comprehension scores from 0 to 6 for each reader in each condition. A 2 (title or no title) $\times 2$ (text: normal or scrambled) repeated measures ANOVA on the comprehension scores revealed a significant main effect of title $[F(1,47)=36$, $\left.M S_{\mathrm{e}}=1.57, p<.01\right]$, indicating significantly better comprehension for titled passages $(3.77,63 \%$ correct $)$ than for passages without titles $(2.68,45 \%$ correct $)$. There was no significant main effect of text, nor was there an interaction between title and text. Thus, scrambling the passages on the second reading did not affect comprehension scores.

Overall, the results of Experiment 1 support the prediction of Raney's (2003) model that changing the text proposition organization upon rereading significantly im- 
pairs transfer when the memory representation is formed with a strong situation model, but not when it is originally formed with a weak situation model. In addition, the results of Experiment 1 offer clear evidence that involvement of the situation model influences not only original reading, but also the reprocessing advantage. Recruitment of the memory representation did not require a situation model, as is indicated by the substantial repetition effect observed in the NT-NT condition. Rather, in Levy (2001) and in Experiment 1, there was an additional transfer advantage when there was also overlap at the level of the situation model, in the T-T, as compared with the NT-NT, condition. These results further support Raney's claim that the total repetition effect is mediated by more than one level of representation.

Experiment 2 tested Raney's (2003) strong prediction concerning transfer across passages. Raney's strong prediction states that when a memory representation is formed around a strong situation model, the surface form and textbase are bound to the text representation and, consequently, repetition benefits will be restricted to similar linguistic contexts. That is, in order for a repetition benefit to occur between passages, there must be overlap at the level of the situation model. We examined Raney's strong prediction by using pairs of highly related and less related passages. The highly related passages were about two different topics (e.g., a passage about New York and a passage about Chicago), but the topics shared a number of themes (e.g., world renowned cities, many attractions to visit, etc.). Thus, there was a great deal of situation model overlap between the passages. The less related passages were about two different topics that did not share themes (e.g., a passage about New York and a passage about the band Pink Floyd). Thus, there was little overlap at the level of the situation model. For both highly related and less related passages, seven sentences were shared by the passages in a pair.

If repetition effects are limited to similar linguistic contexts under reading conditions in which a well-developed situation model is established, as Raney's (2003) strong prediction suggests, processing of the repeated sentences should be facilitated upon rereading in the highly related condition. However, when the passages are less related, according to Raney's strong prediction, there should be little or no facilitation upon the rereading of the repeated sentences because the linguistic contexts are different, so that the situation model formed in the first reading would not be recruited when the sentences are reprocessed in the context of a different situation model.

\section{EXPERIMENT 2}

\section{Method}

Participants. Thirty-five undergraduate volunteers from an introductory psychology course at McMaster University participated in the experiment for course credit. All the volunteers were fluent English speakers.

Materials. In two sessions over 2 days, the participants were presented with four pairs of highly related passages and four pairs of less related passages. Two pairs of each passage type were presented during the first session. In addition, one passage from each of the remaining four pairs was also presented in the first experimental session. This allowed us to create a $24-\mathrm{h}$ interval between passages of a pair. The remaining passages were presented on the 2 nd day. For passage pairs that were presented during the first session, there were always five intervening passages between passage pairs. This was the short-interval condition. In the long-interval condition, one member of a passage pair was presented in the first session, and the second member was presented during the second session. This interval was always $24 \mathrm{~h}$.

All of the passages contained 15-17 sentences and averaged 230 words in length. Of these sentences, 7 were repeated intact in the two passages of a pair. These sentences were between 14 and 17 words in length. The participants controlled the rate of presentation so that they could read at their own pace. The participants pressed a button to begin each passage. The first keypress led to the title of the passage. The next press led to the first sentence of the passage. Passages were presented sentence by sentence down the screen (controlled by the participant's pressing a button), with each new sentence appearing under the previous sentence. Appendix B contains sample passage pairs.

Reading times for each sentence were recorded as the time between successive keypresses and were automatically recorded by the computer. Comprehension questions were given to the participants after the presentation of all the passages, in order to ensure that they were reading for meaning. The questions were not scored for inclusion in data analysis; they were used only as a task orientation instruction. In addition, during the first session, the participants were asked to rate pairs of stories that they had just read. Although passage pairs were intuitively written to be either highly related or less related, in order to verify whether the participants indeed saw them that way, each participant was asked to rate how different the stories were. To cue their memory, the participants were given the titles of each passage in a pair and were asked to think about the passages and then rate how different each pair of stories was on a scale from 0 to 10 . A rating of 0 indicated the story pairs were very similar, and a rating of 10 indicated the story pairs were very dissimilar. The participants were instructed that stories with different themes and different basic topics should be rated toward the higher end of the scale and stories with similar themes and similar basic topics should be rated toward the lower end of the scale.

Procedure. The participants took part in two individual sessions lasting approximately an hour. The participants were told that the experiment was about how people read fluently and that, to that end, they would be presented a number of passages that they were to read fluently - that is, as quickly as they normally would when fluently reading a story. They were told that the passages would appear sentence by sentence on the computer screen, and they were instructed to press a button when they were ready to read and understand each sentence. They were also told that once they started reading a passage, they were not to stop and that they would be asked to answer comprehension questions after reading all of the stories. The participants were then given a practice passage to familiarize them with the sentenceby-sentence presentation and the keypress response. Following this, they were given the experimental passages. When they had finished reading all of the passages, after both sessions, the participants answered the comprehension questions. Finally, in the first session only, the participants rated the passages for semantic similarity.

Design. There were three independent variables: relatedness (highly related or less related), interval (short or long), and repetition (first or second). The times to read the seven sentences that were repeated between passage pairs were combined to yield a total repeated sentence reading time for each member of the pair. The total repeated sentence reading time was then divided by the total number of words in the repeated sentences to yield a measure of word reading speed in the repeated sentences. Consequently, the measure of interest was the time to read each word (in milliseconds) in the repeated sentences. Word reading speed (as opposed to total repeated sentence reading time) was used because it controls for length differences in the repeated sentences. 
Word reading times were first analyzed using a $2 \times 2 \times 2$ repeated measures ANOVA in which relatedness (highly related or less related), interval (short or long), and repetition (first or second) served as the within-subjects variables. In addition, to test for repetition benefits in each condition, $t$ tests were conducted at each level of relatedness by interval: highly related short, highly related long, less related short, and less related long. If repetition benefits are restricted to similar linguistic contexts, processing of the repeated sentences should be facilitated between highly related passages, but not between passages that are less related. Thus, we should find significant repetition benefits in the highly related conditions, but not in the less related conditions, and consequently, a significant interaction between relatedness and repetition.

It was unclear how length of retention interval would affect the repetition benefit. If a longer interval between repetitions significantly reduced the magnitude of the repetition benefit, we should find a significant interaction between interval and repetition and, thus, significantly larger word reading repetition benefits in the short-interval conditions. However, if length of interval did not affect the magnitude of the repetition benefit, we should find comparable repetition benefits across interval conditions.

\section{Results and Discussion}

Table 2 contains the mean word reading times and standard errors for all the conditions. There was a significant interaction between relatedness and repetition $[F(1,34)=$ $\left.7.28, M S_{\mathrm{e}}=791.24, p<.01\right]$. As was expected, this interaction stems from a larger difference between repetitions when the passages were highly related than when the passages were less related. There was also a significant interaction between interval and repetition $[F(1,34)=4.24$, $\left.M S_{\mathrm{e}}=1,275.27, p<.05\right]$. As can be seen from Table 2, this interaction was driven by larger repetition benefits in the short-interval conditions than in the long-interval conditions. There was no significant interaction between relatedness, interval, and repetition $[F(1,34)=0.856$, $\left.M S_{\mathrm{e}}=526.32, p>.05\right]$.

Highly related passages. The words in repeated sentences were read significantly more quickly in highly related passages after both a short interval $[t(34)=7.68$, $p<.001]$ and a long interval $[t(34)=4.1, p<.001]$. Thus, just as Raney's (2003) model would predict, when passages were highly related, there was a significant repetition benefit, and this occurred after both a short and a long interval.

Less related passages. When the passages were less related, the words in repeated sentences were also read

Table 2

Mean Word Reading Times (in Milliseconds) With Standard Errors As a Function of Semantic Relatedness, Interval Between Members of a Passage Pair, and Repetition, Experiment 2

\begin{tabular}{|c|c|c|c|c|c|c|}
\hline \multirow{3}{*}{$\begin{array}{l}\text { Semantic } \\
\text { Relatedness }\end{array}$} & \multicolumn{4}{|c|}{ Repetition } & \multirow{2}{*}{\multicolumn{2}{|c|}{$\begin{array}{c}\text { Repetition } \\
\text { Benefit }\end{array}$}} \\
\hline & \multicolumn{2}{|c|}{ First } & \multicolumn{2}{|c|}{ Second } & & \\
\hline & $M$ & $S E$ & $M$ & $S E$ & $M$ & $S E$ \\
\hline \multicolumn{7}{|c|}{ Five Intervening Passages } \\
\hline Highly related & 289 & 9 & 239 & 9 & 50 & 7 \\
\hline Less related & 280 & 12 & 249 & 10 & 31 & 8 \\
\hline \multicolumn{7}{|c|}{24 Hours } \\
\hline Highly related & 285 & 11 & 254 & 11 & 31 & 7 \\
\hline Less related & 265 & 12 & 251 & 9 & 14 & 8 \\
\hline
\end{tabular}

significantly faster after a short interval $[t(34)=4.37$, $p<.001]$. After a long interval, the repetition benefit was marginal $[t(34)=1.82, p=.08]$. These results do not support Raney's (2003) suggestion that repetition benefits do not cross linguistic boundaries.

Semantic ratings. A rating of 0 indicated that the story pairs were very similar, and a rating of 10 indicated that the story pairs were very dissimilar. A paired sample $t$ test indicated a significant difference in the ratings between the highly related stories and the less related stories $[t(93)=$ $-8.5, p<.001]$. Thus, highly related stories were rated as being more similar (1.98) than less related stories (4.63), although less related passages still received a mean rating below the midpoint of the scale.

The results of this experiment do not support the strong prediction of Raney's (2003) model. When the passages were highly related, the words in repeated sentences were read significantly faster, just as Raney's model would predict. In terms of Raney's model, when the passages were highly related, portions of the surface form and textbase (repeated sentences) were shared between passages that overlapped at the level of the situation model (semantically related). Accordingly, the significant repetition benefit for words in repeated sentences suggests that the memory representation of the first reading episode was recruited to facilitate reading repeated sentences between highly related passages. Thus, just as Raney's model predicts, overlap at the level of the surface form and textbase can produce a repetition benefit if there is also overlap at the level of the situation model.

In less related passages, portions of the surface form and textbase (repeated sentences) were also shared between passages. However, there should be little to no situation model overlap in these passages. The strong prediction of Raney's (2003) model suggests that when there is little semantic overlap between situation models, overlapping surface forms and textbases should produce little to no repetition benefit upon rereading. In this experiment, the words in repeated sentences were read more quickly between less related passages when there was a short interval between repetitions. In addition, when the interval between less related passages was $24 \mathrm{~h}$, there was still a marginal repetition benefit. These results contradict Raney's strong prediction that in the presence of a wellformed situation model, repetition effects will be limited to similar linguistic contexts.

\section{GENERAL DISCUSSION}

The experiments described here explored further the notion that text repetition effects open a window through which we can examine fluency across encounters and the levels of text representation that influence this increasing fluency. Raney's (2003) wedding of repetition effects with the text representation model of van Dijk and Kintsch (1983) raises important issues regarding the way knowledge is stored when texts are read and how these representations can be recruited to aid later processing. In both experiments, we examined the notion of contextual dependency of the repetition benefit. 
Experiment 1 replicated the major finding of Levy (2001) showing that memory representations, including representations at the level of the situation model, influence the magnitude of the reprocessing benefit, consistent with Raney's (2003) model. In addition, the results of Experiment 1 supported Raney's notion that when text is read with a well-developed situation model, it becomes bound to the original situation model. Scrambling the sentence order in the second reading created a mismatch between the text-propositional representation from the first reading and the text-propositional representation from the second reading. This mismatch led to a significantly smaller repetition benefit in the presence of a situation model ( $\mathrm{T}-\mathrm{TS}$ condition), but not when a situation model was absent (NT-NTS condition).

In Experiment 2, we found a significant repetition benefit between less related passages. These results are incompatible with the strong prediction of Raney's (2003) model, in which the textbase and surface form are bound to the situation model, thereby limiting repetition effects to similar linguistic contexts. Rather, taking into consideration the participants' semantic ratings of the passages, as well as the overlapping textbase representations between passage pairs, the pattern of results in these experiments support better a context-dependent continuum explanation (Raney, 2003). In a context-dependent continuum, the more overlap between two texts at the level of the surface form, textbase, and situation model, the more processing should be facilitated between the texts. In Experiment 2, the participants rated pairs of highly related passages as being more similar than less related passages. However, less related passages were not rated as completely dissimilar, possibly because of the seven overlapping sentences. Thus, in regards to a context-dependent continuum, a significant repetition benefit between less related passages would not be totally unexpected. The results revealed that smaller repetition benefits corresponded with a semantic rating indicating small amounts of semantic relatedness. Similarly, when passages were rated as being highly similar, there were larger repetition benefits. Of course, in order to definitively argue for the existence of a contextdependent continuum, it will be necessary in future research to consider three or more levels of the continuum to show that the magnitude of the repetition benefit can vary depending on the degree of overlap.

The results of Experiment 1 are also consistent with a context-dependent continuum explanation. This is due to the nature of the passages in the experiment. It is difficult to understand what each passage is about without a title, regardless of reading ability. Thus, it is conceivable that processing of the passages with a title leads to a text representation that falls near the context-dependent end of a continuum. Processing of the passages without a title, on the other hand, probably leads to a representation more toward the context-independent end of a continuum, since it is essentially a case of reading without comprehension. Overall, then, the conditions that affect situation model development (task demands, processing strategies, text coherence, and reader characteristics) are set up to produce representations at either end of the context- dependent continuum, depending on whether a title is presented or not. As a result, when the passages are presented with a title, the lower levels will appear to be bound, when in fact, the representation of the text is simply easily disrupted by any change upon rereading because it falls close to the context-dependent end of the continuum.

If we are to accept the notion of a context-dependent continuum, whereby the greater the similarity between the situation models of two texts, the larger the expected repetition effect, questions about the criteria for defining overlapping situation models raised by the results of Experiment 2 need to be answered. Pairs of passages in the less related conditions were about very different topics. However, the participants instructed to rate the passages on the basis of their underlying topics and themes still did not see the passages as being completely dissimilar. This could have been due to the number of overlapping sentences between passages, rather than to shared conceptual ideas. Consequently, the question of what criteria are used to define overlapping and nonoverlapping situation models needs clarification. It seems unlikely that participants would produce the same ratings if less obvious elements of the text representation (such as repeated words or font) were shared between passages. Perhaps it is more precise to say that these ratings are indicative of a difficulty in distinguishing between textbase representations and situation model elements. If this is the case, it is not overlapping situation models that determine the magnitude of the repetition effect. Instead, larger repetition benefits would be expected with greater similarities between the situation model and the textbase of passages.

The findings of these experiments provide little support for either a strictly abstract or a strictly episodic account of text repetition effects. In the experiments reported here, repetition effects were largest when there was overlap at the level of the situation model. This illustrates the important role context plays in determining the magnitude of the repetition benefit. In other words, it seems that overlap at the level of the situation model provides a mechanism by which surface features and textbase elements enhance repetition effects in the context of a well-developed situation model. This is incompatible with a strictly abstract account. In addition, we found repetition benefits when there was incomplete overlap at the level of the situation model. This illustrates that surface form and textbase representations can also facilitate processing of a new text. A strictly episodic account of text repetition effects cannot explain these findings.

Overall, the experiments reported here suggest that Raney's (2003) model can make a substantial contribution to explanations of how texts are represented in memory and how these representations can be recruited to the services of increased reading fluency. Experiments 1 and 2 illustrate how the three levels of text representation outlined in Raney's model influence the way texts are represented in memory and determine the magnitude of subsequent repetition effects. The results of these experiments provide preliminary support for the idea that the degree of overlap at each level of representation determines how much of a repetition benefit there is between passages. 
However, the notion of a context-dependent continuum and the question of how it affects fluent reading still need further investigation.

\section{AUTHOR NOTE}

This research was supported by an operating grant from the Natural Sciences and Engineering Research Council of Canada to the second author. The authors thank Ken McRae, Tom Carr, and two anonymous reviewers for their helpful comments. Correspondence concerning this article should be addressed to W. M. Collins, Department of Psychology, Neuroscience, and Behaviour, McMaster University, 1280 Main St. W., Hamilton, ON, L8S 4K1 Canada (e-mail: collinwm@mcmaster.ca).

\section{REFERENCES}

BRANSFORD, J. D. (1979). The role of prior knowledge. In J. D. Bransford (Ed.), Human cognition: Learning, understanding and remembering (pp. 129-165). Belmont, CA: Wadsworth.

BROWN, J. S., \& CARR, T. H. (1993). Limits on perceptual abstraction in reading: Asymmetric transfer between surface forms differing in typicality. Journal of Experimental Psychology: Learning, Memory, \& Cognition, 19, 1277-1296.

Carlson, L., Alejano, A., \& Carr, T. H. (1991). The level of focal attention hypothesis in oral reading: Influences of strategies on the context specificity of lexical repetition effects. Journal of Experimental Psychology: Learning, Memory, \& Cognition, 17, 924-931.

CARR, T. H., \& BRown, J. S. (1990). Perceptual abstraction and interactivity in repeated oral reading: Where do things strand? Journal of Experimental Psychology: Learning, Memory, \& Cognition, 16, 731-738.

Carr, T. H., Brown, J. S., \& Charalambous, A. (1989). Repetition and reading: Perceptual encoding mechanisms are very abstract but not very interactive. Journal of Experimental Psychology: Learning, Memory, \& Cognition, 15, 763-778.

LEVY, B. A. (1993). Fluent reading: An indirect indicator of reading skill development. In P. Graf \& M. E. J. Masson (Eds.), Indirect mem- ory: New direction in cognition, development, and neuropsychology (pp. 49-73). Hillsdale, NJ: Erlbaum.

LEVY, B. A. (2001). Text processing: Memory representations mediate fluent reading. In M. Naveh-Benjamin, M. Moscovitch, \& H. L. Roediger III (Eds.), Perspectives on human memory and cognitive aging: Essays in honour of Fergus Craik (pp. 83-98). New York: Psychology Press.

Levy, B. A., \& Burns, K. I. (1990). Reprocessing text: Contributions from conceptually driven processes. Canadian Journal of Psychology, 44, 465-482.

Levy, B. A., Campsall, J., Browne, J., Cooper, D., Waterhouse, C., \& Wilson, C. (1995). Reading fluency: Episodic integration across texts. Journal of Experimental Psychology: Learning, Memory, \& Cognition, 21, 1169-1185.

Levy, B. A., DiPersio, R., \& Hollingshead, A. (1992). Fluent reading: Repetition, automaticity, and discrepancy. Journal of Experimental Psychology: Learning, Memory, \& Cognition, 18, 957-971.

LeVy, B. A., \& Kirsner, K. (1989). Reprocessing text: Indirect measures of word and message level processes. Journal of Experimental Psychology: Learning, Memory, \& Cognition, 15, 407-417.

Levy, B. A., Masson, M. E., \& Zoubeck, M. A. (1991). Rereading text: Words and their context. Canadian Journal of Psychology, 45, 492-506.

RANEY, G. E. (2003). A context-dependent representation model for explaining text repetition effects. Psychonomic Bulletin \& Review, 10, 15-28.

Raney, G. E., Minkoff, S., \& Therriault, D. (1997, November). Using interleaved reading to study text processing. Paper presented at the 38th Annual Meeting of the Psychonomic Society, Philadelphia.

Raney, G. E., Therriault, D., \& Minkoff, S. (2000). Repetition effects from paraphrased text: Evidence for an integrated representation model of text representation. Discourse Processes, 29, 61-81.

Tenpenny, P. L. (1995). Abstractionist versus episodic theories of repetition priming and word identification. Psychonomic Bulletin \& Review, 2, 339-363.

VAN DiJK, T. A., \& KinTsch, W. (1983). Strategies of discourse comprehension. New York: Academic Press.

\section{APPENDIXA \\ A Normal (Title Below in Parenthesis) and Scrambled Passage With Sample Comprehension Questions, Experiment 1}

The first part of this process involves driving to a certain location. Walking is not a suitable alternative. Once one arrives, it is important to present documentation. Most people will have prepared such papers in advance in order to make the process as hassle free as possible. An efficient entrance into the facility is preferred as the person involved in this event has little time to spare, yet a lot of work to accomplish. The flood of emotions experienced during the process is perhaps greater incentive to preserve the individual's patience. While anticipation may dominate, this feeling is often intermingled with ample frustration and in some cases even agony. Such emotional turmoil is attributed to a variety of causes. First, tempers may flare as a direct result of the differential distribution of the workload during the procedure. While many individuals are present as observers throughout the process, only one person bears the full burden of responsibility. Ironically, this person is generally the least experienced with the process. Further, the novice is often significantly impaired during the latter stages of the procedure. This practice is perhaps one of the only legal processes in which the individual with the most responsibility is permitted to be fully inebriated. The observers, in contrast, have participated in the event many times. They take absolutely no share, however, in the actual physical work done. Instead, they are present only to provide instruction for the novice. Further, they must be completely sober throughout the entire activity. While the separation of responsibility in this process appears unfair, balancing the workload among all individuals present is, unfortunately, impossible. The novice may also be annoyed with the observers due to the nature of their instructions. Often, their orders are quite inconsistent. Initially, they will demand that the person not do something. Later in the procedure, however, they will demand that the person engage in the formerly forbidden task. Finally, the person's anger may be heightened considering that the observers are given unrestricted access to personal space. Similar to the aforementioned causes of frustration, this practice is unavoidable. In the event that the process does not progress smoothly, the observers can be of assistance. In such circumstances their role is actually reversed from that described above. For example, these individuals take on the majority of responsibility and effort. While it seems counter-intuitive, most participants still prefer to shoulder the full workload. 


\section{APPENDIXA (Continued)}

(Having a Baby)

1. What must be prepared in advance in order to make entrance into the facility as hassle-free as possible?

2. Who bears the burden of responsibility and the majority of the workload in this procedure?

3. What are the observers given unrestricted access to?

The first part of this process involves driving to an appropriate location. Only one central person bears the full burden of responsibility. Such emotional turmoil is attributed to a variety of causes. They take absolutely no share, however, in the actual physical work done. While anticipation may dominate, this feeling is often intermingled with frustration and in some cases even agony. In such circumstances their role is actually reversed from that described above. Walking is not a suitable alternative. Later in the procedure they will demand that the person engage in the formerly forbidden task. An efficient entrance into the facility is preferred. For example, these individuals take on the majority of responsibility and effort. Similar to the aforementioned causes of frustration, this practice is unavoidable. The separation of responsibility in this process appears unfair. The person involved in this event has little time to spare with a lot of work to accomplish. First, tempers may flare as a direct result of the differential distribution of the workload during the procedure. Preparing appropriate documentation makes the process as hassle free as possible. Ironically, this person is generally the least experienced with the process. In the event that the process does not progress smoothly, the observers can be of assistance. Once one arrives, it is always important to present documentation. The flood of emotions experienced during the process is perhaps greater incentive to preserve the individual's patience. The novice may also be annoyed with the observers due to the nature of their instructions. Instead, they are present only to provide instruction for the novice. Most people will have prepared such papers in advance. Balancing the workload among all individuals present is, unfortunately, impossible. This practice is one of the only legal processes in which the individual with the most responsibility is permitted to be fully inebriated. Many individuals are present as observers throughout the process. This may be because the observers are given unrestricted access to personal space. Often, their orders are quite inconsistent. Further, the novice is often significantly impaired during the latter stages of the procedure. While it seems counter-intuitive, most participants still prefer to shoulder the full workload. Finally, the person's anger may be heightened. The observers, in contrast, have usually participated in the event many times. Initially, they will demand that the person not do something. Further, they must be completely sober throughout the entire activity.

APPENDIX B

Highly Related (The Second City and The Big Apple) and Less Related (The Big Apple and Pink Floyd) Passage Pairs, Experiment 2 (Repeated Sentences in Bold)

\section{The Second City}

Chicago is the major hub in the American Midwest.

It is a major business center and attracts millions of tourists each year.

It is so memorable, once you experience it, you will not forget it anytime soon.

The city is filled with many museums to visit and almost every month there is a street festival to experience. Chicago is the birthplace of the Blues and hosts the largest Blues festival in the world.

As you would expect with such an event, the area is filled with crowds of people.

It is a diverse crowd made up of people with music as the common interest.

Despite all the other attractions around the area, everyone is there to enjoy the music.

Many tourists visit the Museum of Science, home of the first OmniMax theatre.

The area is set up with giant video screens that show films for a complete multisensory experience.

The museum contains the Apollo 8 space capsule which orbited the moon.

The city is also home to the Lincoln Park Zoo which has free admission and markets animal shaped balloons.

Many others have tried to copy this idea but have had limited success in doing so.

Gigantic balloons of various animals can be seen floating in the air from miles away.

A popular destination, Chicago is behind only Las Vegas and Orlando in the number of conventions it hosts annually.

This is probably due to the large number of companies and universities in the area.

For example, Chicago is home to seven medical schools and a number of leading healthcare organizations.

\section{The Big Apple}

New York is one of the largest cities in the United States.

There are so many things to see and do if you visit the city.

It is so memorable, once you experience it, you will not forget it anytime soon.

In addition to popular attractions like the Statue of Liberty, there are many smaller festivals including the Bronx Jazz festival. 


\section{APPENDIX B (Continued)}

The streets are filled with vendors and sponsors trying to market their products.

As you would expect with such an event, the area is filled with crowds of people.

It is a diverse crowd made up of people with music as the common interest.

Despite all the other attractions around the area, everyone is there to enjoy the music.

The Tribeca film festival also attracts many visitors in the spring.

The area is set up with giant video screens that show films for a complete multisensory experience.

Many big stars come out for the festival including Tribeca film festival founder and neighbourhood resident Robert De Niro.

One of the many trademarks of the city is the Macy's Thanksgiving Day Parade.

Many others have tried to copy this idea but have had limited success in doing so.

Gigantic balloons of various animals can be seen floating in the air from miles away.

Prior to 1927, real animals borrowed from the Central Park Zoo were used in the parade.

People often forget about the many other attractions New York has to offer.

This is a shame as these events provide real insight into what the city is really like.

\section{Pink Floyd}

Pink Floyd was one of the most popular progressive rock bands.

They were known for both philosophical lyrics and experimentation but especially for their elaborate live shows.

It is so memorable, once you experience it, you will not forget it anytime soon.

At one time, Pink Floyd held the record for the largest grossing tour of all time.

Their concerts were filled with special effects including video, lasers, lights and quadraphonic speaker systems.

As you would expect with such an event, the area is filled with crowds of people.

It is a diverse crowd made up of people with music as the common interest.

Despite all the other attractions around the area, everyone is there to enjoy the music.

It is rather incredible how the band pulls off these concerts.

The area is set up with giant video screens that show films for a complete multisensory experience.

A crew of over 150 people is hired for the pyrotechnics such as an exploding gong.

The band first introduced the use of helium balloons during a tour in 1975 and expanded their use during the

'Animals' tour.

Many others have tried to copy this idea but have had limited success in doing so.

Gigantic balloons of various animals can be seen floating in the air from miles away.

Even more inflatables were used as giant string puppets during tours for 'The Wall'.

Unfortunately, the music has often been overshadowed by the special effects.

However, the music has always been critically acclaimed in its own right. 